\title{
Development of a New Closed-type Environmental-cell Specimen Holder with High Gas Conductivity
}

\author{
T. Kawasaki***, T. Miura*, H. Tsutsui***, T. Matsutani*** and T. Tanji**** \\ * $\quad$ Dept. Electrical Eng., Nagoya Univ., Furo-cho, Chikusa-ku, Nagoya, 464-8603, Japan \\ ** PRESTO, Japan science and Technology Agency, 4-1-8 Honcho Kawaguchi, Saitama, Japan \\ *** Graduate School of Engineering, Kinki Univ., 3-4-1Higashiosaka, Osaka, 577-8502, Japan \\ **** EcoTopia Science Inst., Nagoya Univ., Furo-cho, Chikusa-ku, Nagoya, 464-8603, Japan
}

In-situ controlled-environment TEM (ETEM) is a powerful technique to study nature of materials under the conditions in which they are formed or applied. In cases of actual materials such as catalysts, fuel cells, biological molecules, etc., it is essential to obtain their practical information, not that in laboratory. For this purpose, ETEM has been investigated during about half century $[1,2]$. The authors has been also developed a closed-type ETEM system with windowed environmental-cell (E-cell) [3,4]. The E-cell systems utilize the dedicated specimen holder which enables to introduce gases or liquids around specimens and to seal them with thin films. The biggest advantage of this type is to realize the real conditions in the TEM, where the specimens are applied actually, for example, atmospheric pressure, wet environment, and so on [3-8]. However, the conventional closed-type systems have a disadvantage that smooth gas flow is difficult because of small gas pipes assembled in the specimen holder. To solve this problem, we developed a new E-cell specimen holder having higher gas conductivity [9].

FIG. 1 shows schematic diagrams of the E-cell specimen holders with simple functions; gas introduction and evacuation. In the conventional specimen holder (left-hand side), two pipes connected toward the E-cell are assembled for the above two functions. Therefore, the diameter of each pipe is restricted up to a few mm. Such narrow passes disturb smooth gas flow and result in difficulty of gas pressure control inside the E-cell. The right-hand side of the FIG. 1 is our developed E-cell specimen holder, named "coaxial line type". Here, just one pipe is set for gas introduction and the residual space in the holder shaft is used for gas evacuation. The pipe size is then allowed to be bigger than that in the conventional ones. FIG. 2 shows pictures of the coaxial line type E-cell specimen holder, dedicated for the TEM; H-8000 (Hitachi). In our case, the inner diameter of the pipe was expanded more than 3 times comparing with the previous E-cell specimen holder, resulting in 10 times improvement of the gas conductivity.

\section{References}

[1] H. Hashimoto, J. Electron Microsc. 9, 130 (1960).

[2] H. G. Heide, Die Naturwissenschaften 14, 313 (1960). 
[3] K. Ueda et al., Surf. Interface Anal. 40, 1725 (2008).

[4] T. Kawasaki et al., Rev. Sci. Inst. 80 (2009) 113701

[5] A. Ishikawa et al., J. Electron Microsc. 38, 316 (1989).

[6] P. L. Gai, Top. Catal. 21, 161 (2002).

[7] S. Giorgio et al., Ultramicroscopy 106, 503 (2006).

[8] J. F. Creemer et al., Ultramicroscopy 108, 993 (2008).

[9] T. Kawasaki, Japanese Patent No. 2008-226466
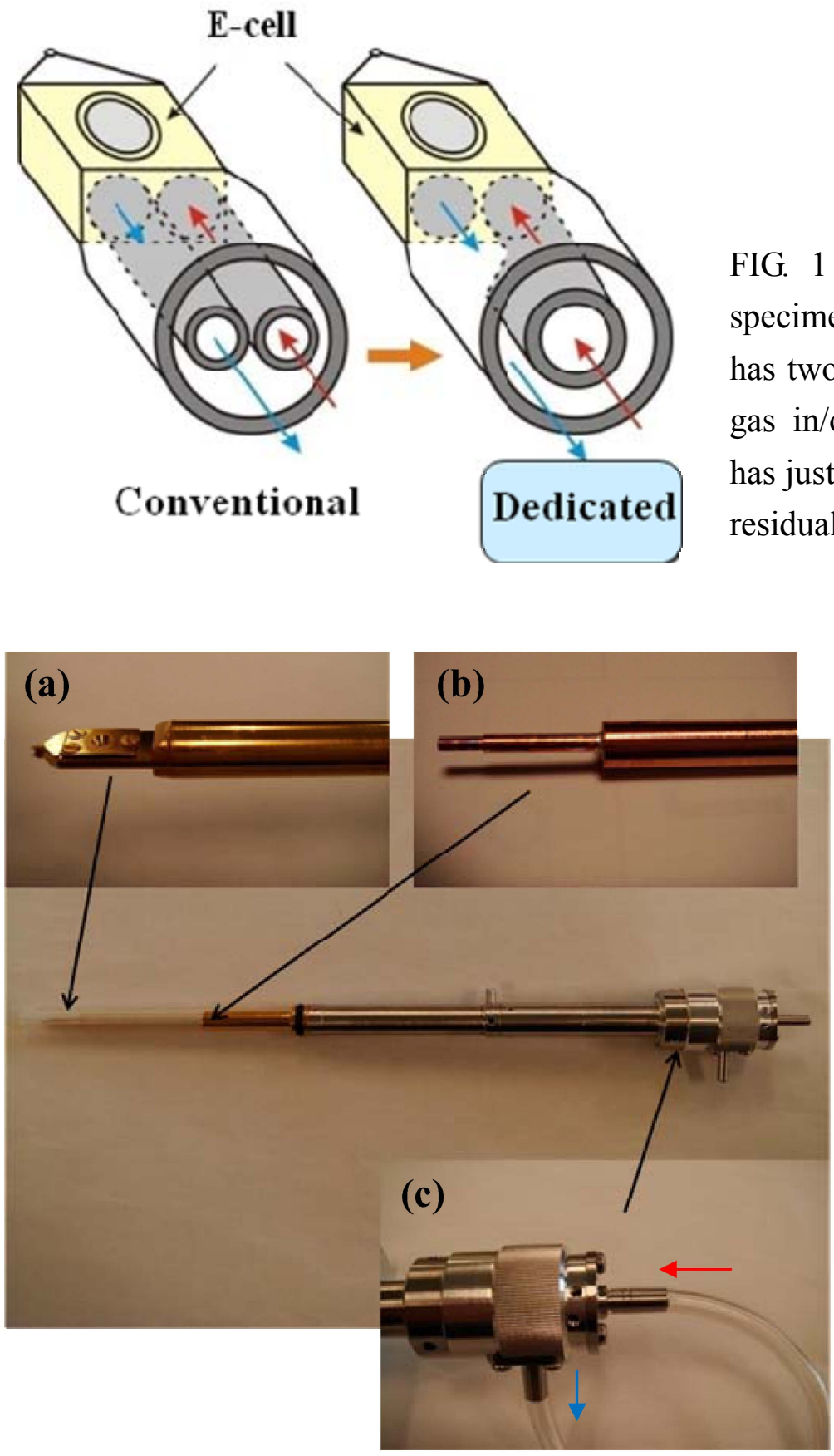

FIG. 1 Schematic diagrams of E-cell specimen holders. Left; conventional type has two pipes connected to the E-cell for gas in/out. Right; new dedicated holder has just one pipe for gas introduction, and residual space is used for its evacuation.
FIG. 2 Pictures of coaxial line type E-cell specimen holder. (a) E-cell part; (b) a pipe for gas introduction; (c) gas tube connecters. 